\title{
The importance of quantitative Mössbauer spectroscopy of MoFe-protein from Azotobacter vinelandii
}

\author{
William R. DUNHAM, Wilfred R. HAGEN, Arnold BRAAKSMA, Hans J. GRANDE and Huub HAAKER \\ Biophysics Research Division, Institute of Science and Technology, The University of Michigan, Ann Arbor; \\ Department of Biochemistry, Agricultural University, Wageningen; and \\ Institute of Applied Chemical Research (TNO-ITC), Zeist
}

(Received June 8/September 28, 1984) - EJB 840606

\begin{abstract}
The Mössbauer spectra of MoFe-protein of Azotobacter vinelandii, as isolated under dithionite and taken at temperatures from $125 \mathrm{~K}$ to $175 \mathrm{~K}$, are the sums of four resolved quadrupole doublets. Our results indicate that the currently accepted interpretation of these doublets can be questioned. Our data reduction method converts the Mössbauer transmission spectra to source lineshape deconvolved absorption spectra linear in iron. We used these absorption spectra to determine the stoichiometry of the Fe clusters in MoFe-protein and we obtained much better fits if we assumed that there are four iron atoms in the ' $\mathrm{Fe}^{2+}$ ' doublet, two iron atoms in the ' $\mathrm{S}$ ' doublet, twelve iron atoms in the ' $D$ ' doublet and sixteen iron atoms in the ' $M$ ' doublet. Therefore we propose that the MoFe-cofactor contains one molybdenum and eight iron atoms ('M').

We also argue that none of the previous Mössbauer spectroscopic studies have been performed on the highestactivity preparation now obtainable, nor has there been any study to prove that the Mössbauer spectra are independent of activity. We consider that the Mössbauer spectroscopic studies of the MoFe-protein of nitrogenase are a re-opened and unsolved problem.
\end{abstract}

The determination of the transition metal distribution within the MoFe-protein component of nitrogenase has largely been the result of Mössbauer spectroscopic experiments on samples from Klebsiella pneumoniae [1] and Azotobacter vinelandii [2]. The purpose of this paper is to demonstrate a weakness in these interpretations, to offer an alternative explanation, and to outline the method by which we feel this problem should be solved. By the latter, we mean that not only are the Mössbauer spectra for the MoFe-protein susceptible to large distortions during data reductions, but also that the samples that were used in the previous studies are not of the highest activity achieved in today's experiments, and are, therefore, not necessarily representative of the true metal content of the MoFe-protein in vivo.

The nitrogenase complex consists of two protein units: the $\mathrm{MoFe}$-protein and the Fe-protein. The MoFe-protein $\left(M_{\mathrm{r}}=220000\right)$ has two subunits of $50 \mathrm{kDa}$ and two subunits of $60 \mathrm{kDa}$. It contains the active site for the reduction of dinitrogen; fully active MoFe-protein contains 2 Mo atoms and $24-32 \mathrm{Fe}$ and $\mathrm{S}$ atoms per complex $[3,4]$. The Fe-protein is a dimer $\left(M_{\mathrm{r}}=63000\right.$ total $)$; it reduces the MoFe-protein in the presence of MgATP, and it is reported to have four Fe and four $\mathrm{S}$ atoms per dimer [3] but recent studies indicate that higher contents are possible [5]. MoFe-protein has been

Abbreviations. The MoFe and Fe-proteins of the nitrogenases of Azotobacter vinelandii, Clostridium pasteurianum and Klebsiella pneumoniae are referred to as $A v_{1}$ and $A v_{2}, C p_{1}$ and $C p_{2}$ and $K p_{1}$ and $\mathrm{Kp}_{2}$ respectively, $\mathrm{Av} \mathrm{Fe} / \mathrm{S}$ II stands for $\mathrm{Fe} / \mathrm{S}$ protein II from $A$. vinelandii; EPR, electron paramagnetic resonance; ENDOR, electron nuclear double resonance.

Enzyme. Nitrogenase (EC 1.18.2.1). studied by EPR and Mössbauer spectroscopies. Smith et al. $[1,6]$ studied $\mathrm{Kp}_{1}$ and $\mathrm{Kp}_{2}$ and Münck et al. did an extensive study on $\mathrm{Av}_{1}[2,7,8]$ and $\mathrm{Cp}_{1}[9]$. From those studies, it was proposed that the MoFe-protein has two FeMo-cofactors $[1 \mathrm{Mo}-6 \mathrm{Fe}-6 \mathrm{~S}]$, four $\mathrm{P}$ clusters $[4 \mathrm{Fe}-4 \mathrm{~S}]$ and two iron atoms in an unknown arrangement, possibly in a $[2 \mathrm{Fe}-2 \mathrm{~S}]$ cluster [6]. The FeMo-cofactor has been isolated from the MoFeprotein [10] and studied by Mössbauer spectroscopy [11]. A recent determination of the stoichiometry of the MoFecofactor has been performed by a radiochemical method. The observed stoichiometry is $1 \mathrm{Mo}: 8.2 \mathrm{Fe}: 8.7 \mathrm{~S}$ [12]. In the presence of dithionite, preparations of $\mathrm{Av}_{1}$ show an EPR signal that has been attributed to an $S=3 / 2$ spin state of the FeMo-cofactor [2]. ENDOR studies of the dithionite-reduced MoFe-protein have assigned the Mo atom to the $S=3 / 2$ signal [13]. Furthermore, recent ENDOR results have tentatively identified six unique iron sites, with the projections of their magnetic hyperfine tensors along the principal axes of the $S=3 / 2 \mathrm{~g}$-tensor [14]. The substitution of these values into a Mössbauer spectroscopic synthesis program (Hoffman, B. M., personal communication) appears to result in good fits and to confirm the previous assignments by the Mössbauer spectroscopists $[2,7-9]$.

For the purposes of this article, it is necessary to discuss only the high-temperature $(125-175 \mathrm{~K})$ Mössbauer spectra of the MoFe-protein (as isolated) from Azotobacter vinelandii $\left(A v_{1}\right)$. These Mössbauer spectra are comprised of four quadrupole pairs. We will use the previously defined $[2,15]$ nomenclature of these pairs: ' $M$ ' for the iron atoms in the FeMo-cofactor, ' $\mathrm{D}$ ' for the larger iron component of the $\mathrm{P}$ clusters; ' $\mathrm{Fe}^{2+}$ ' for the smaller iron component of the $\mathrm{P}$ clusters; and ' $\mathrm{S}$ ' for the remaining doublet in the spectra. The 
question of central importance is: How many iron atoms are represented by each doublet?

\section{MATERIALS AND METHODS}

\section{Growth conditions and enzyme preparation}

Azotobacter vinelandii ATCC 478 was grown in the standard Burk's nitrogen-free basic salt medium in a batch culture as described earlier [5] except that $\mathrm{FeSO}_{4} \cdot 7 \mathrm{H}_{2} \mathrm{O}$ was replaced by $1 \mathrm{mg} / \mathrm{ml}{ }^{57} \mathrm{Fe}$.

Isolation of the nitrogenase proteins and standard nitrogenase activity assays were run as described earlier [5]. $A v_{1}$ and $A v_{2}$ purified by the described procedure had specific activities of $2000-2400 \mathrm{nmol} \mathrm{C}_{2} \mathrm{H}_{4}$ formed $\cdot \min ^{-1}$ - (mg MoFe-protein $)^{-1}$ and $1800-2000 \mathrm{nmol} \mathrm{C}_{2} \mathrm{H}_{4}$ formed $\cdot \min ^{-1} \cdot(\mathrm{mg} \text { Fe-protein })^{-1}$. The activity of MoFe-protein was not enhanced when its activity was measured with Fe-protein with more than four $\mathrm{Fe}$ atoms and four sulfide atoms/molecule [5]. The MoFe-protein used in this study had a specific activity of $2250 \mathrm{nmol} \mathrm{C}_{2} \mathrm{H}_{4}$ formed $\cdot \mathrm{min}^{-1} \cdot \mathrm{mg} \mathrm{Av}_{1}^{-1}$. The $\mathrm{Mo}, \mathrm{Fe}$ and $\mathrm{S}^{2-}$ content per $\mathrm{mg} \mathrm{Av} \mathrm{v}_{1}$ was $7.3 \pm 0.4 \mathrm{nmol} \mathrm{Mo}$, $109 \pm 9 \mathrm{nmol} \mathrm{Fe}$ and $100 \pm 9 \mathrm{nmol} \mathrm{S}{ }^{2-}$. The molar ratio of $\mathrm{Mo}: \mathrm{Fe}: \mathrm{S}^{2-}$ per molecule $\mathrm{Av}_{1}$ is $1.6: 24: 22$.

\section{Analytical methods}

The Mössbauer spectra were taken on a previously described spectrometer [16] with a Cryogenics Associates dewar and a highly modified Ranger Instruments drive. We have recently added a type II servo mechanism to the feedback loop of the drive [17]. Further papers characterizing the servo loop performance and design are presently in preparation. The drive routinely demonstrates an accuracy and precision of less than $\pm 0.001 \mathrm{~mm} / \mathrm{s}$ over the entire span of a $24-\mathrm{h}$ experiment. We know of no other spectrometer that can match this short or long-term stability performance specification. Furthermore, the long-term stability is also demonstrated by the $125 \mathrm{~K}$ spectra in this article, whose data were collected over a six-week period due to the low count rate from our 2-year-old 35 -mCi ${ }^{57} \mathrm{Co}$-in-rhodium source.

Molybdenum [18], iron [19] and sulphide [20] were determined using published procedures. Protein concentration was estimated with the micro-biuret method of Goa [21] with bovine serum albumin as the standard.

\section{Materials}

ATP, creatine kinase (EC 2.7.3.2), creatine phosphate, and bovine serum albumin were obtained from Sigma Chemical $\mathrm{Co}$; all other chemicals were of the highest commercial grade. Argon was purified by passage over hot $\left(150^{\circ} \mathrm{C}\right) \mathrm{BASF}$ catalyst.

\section{RESULTS}

Fig. 1A shows the raw data from a $125 \mathrm{~K}$ Mössbauer spectrum of $A v_{1}$ corrected only for pulse pile-up and solid angle effects [16]. It is, therefore, suitable for comparison with data from other $\mathrm{MoFe}$-protein preparations. If the background counts due to non-resonant $14-\mathrm{keV}$ gamma rays and Compton events in the gas counter are subtracted, Fig. $1 \mathrm{~B}$ is obtained with an increase in the percentage effect from the background correction. If then the spectrum is deconvolved by the source lineshape [16], Fig. 1C results again with a significant increase in the percentage effect. The mathematical process that performs this deconvolution requires a truncation in Fourier space; therefore, there are ripples in the original space version of this spectrum (Fig. 1C). Others $[8,9]$ have also used Fourier deconvolution on their spectra, but the method of Ure and Flinn [22] used by these authors is imprecise and results in an error that makes it useless in the following step.

In this step, the logarithm of the deconvolved data was taken, giving the result shown in Fig. 1D. Note that the maxima are well into the region where $\mathrm{e}^{-x} \neq 1-x$. Thus, data treatments that ignore this step have large line distortions. The Fourier deconvolution method of Ure and Flinn [22] also should give a spectrum corresponding to Fig. 1D. However, in that method, the Fourier deconvolution step is stabilized in Fourier space with multiplication by a Gaussian lineshape. This procedure is an explicit convolution, whose side effect is that it prevents one from accurately taking the logarithm of the data in the following step. The process of taking the logarithm of a function to be Fourier-transformed is mathematically complicated [23]. In order to stabilize the Fourier space one needs to set to zero those values where the deconvolved noise in the Fourier space is much larger than the deconvolved signal by multiplying them by a 'filter' function. Any convex function can be made to act as a filter function including the Gaussian function. On the other hand, one needs to leave unchanged those values in Fourier space where the signal/noise ratio is higher than unity, so that taking the logarithm of the backtransformed spectrum has the maximum chance to generate a valid representation of the signal. Truncation multiplies by one those elements in Fourier space where signal/noise is greater than one and multiplies by zero those elements where it is less than one. Truncation is, therefore, the ideal filter. The method of Ure and Flinn requires the use of a filter function that greatly modifies some of the significant elements in Fourier space and at least weakly modifies all the elements of the Fourier space so that their method can be much less accurate than our method $[16,17$, 24]. Furthermore, our minimization (curve-fitting) procedures take place in the Fourier space so that they are almost unaffected by the truncation.

In Fig. 2A, the backtransform is shown of one of these fits superimposed on the data with the difference spectrum below (Fig. 2B). If the 'ripples' in the data and simulation are bothersome, we suggest that one concentrate on the difference spectrum where these ripples are absent. (The periodic nature of the difference spectrum also results from truncation in Fourier space, but these ripples are due to 'noise' if the fit is perfect.) In the fit, as shown in Fig.2A, we allow the isomer shifts and quadrupole splittings of four independent quadrupole pairs to vary until a best fit is obtained, while fixing the relative intensities of the four pairs to the following ratio: $4: 2: 12: 12$. This is the currently accepted model for the MoFe-protein: 4 iron atoms in center ' $\mathrm{Fe}^{2+} ; 2$ iron atoms in ' $\mathrm{S}$ '; 12 iron atoms in ' $\mathrm{M}$ '; and 12 iron atoms in ' $\mathrm{D}$ '. The fit is not bad, but it is much worse than the fit shown in Fig. 2C. The difference spectrum is shown in Fig. 2D. In this fit, the iron ratios are $4: 2: 16: 12$.

In Fig. 3 the data and fits with ratios $4: 2: 16: 12$ for $150 \mathrm{~K}$ and $175 \mathrm{~K}$ are shown. All the lines in these simulations have the same linewidth (FWHM) of $0.16 \mathrm{~mm} / \mathrm{s}$. The lineshape should be the convolution of a Heissenberg Lorentzian lineshape and the frequency function for the Mössbauer 


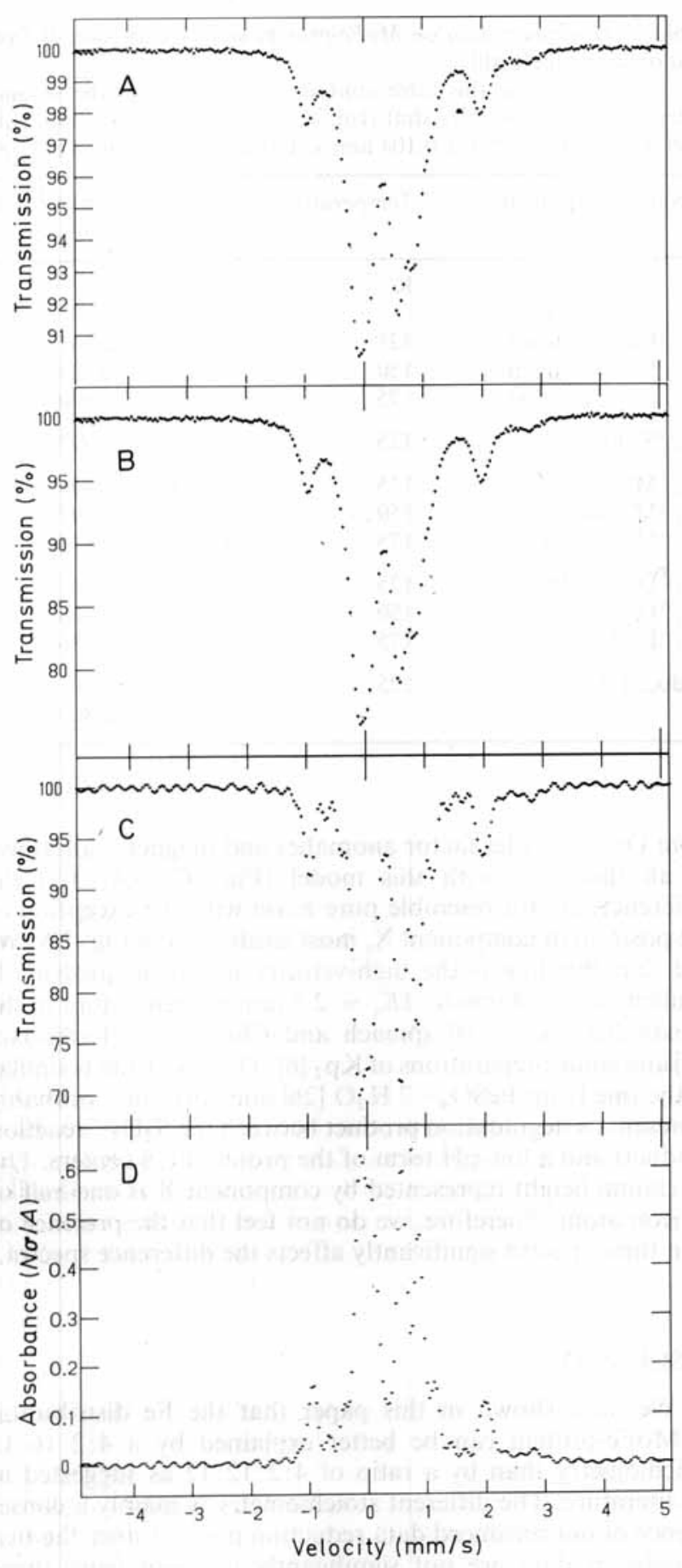

Fig. 1. Mössbauer data for $A v_{1}$ as isolated, at $125 \mathrm{~K}$ and zero applied magnetic field. The isomer shifts are relative to iron metal at $298 \mathrm{~K}$ and corrected to a $298 \mathrm{~K}$ standard absorber state using the Debye model with $\Theta_{\mathrm{D}}=180 \mathrm{~K} . \mathrm{Av}_{1}(21.9 \mathrm{mg} / \mathrm{ml})$ was isolated as described in Materials and Methods. (A) Raw data, corrected for pulse pile-up and solid angle effects. (B) Data corrected for background due to nonrecoil-free gamma rays in the beam and Compton events in the counter. (C) Background-corrected data deconvolved by the source lineshape. (D) Background-corrected and deconvolved data, plotted as logarithmic values

parameters distribution. The use of a Lorentzian lineshape, although not formally correct, does not result in an error larger than the noise in this experiment when the width is this small $(0.16 \mathrm{~mm} / \mathrm{s})$. The $4: 2: 16: 12$ simulations have the same

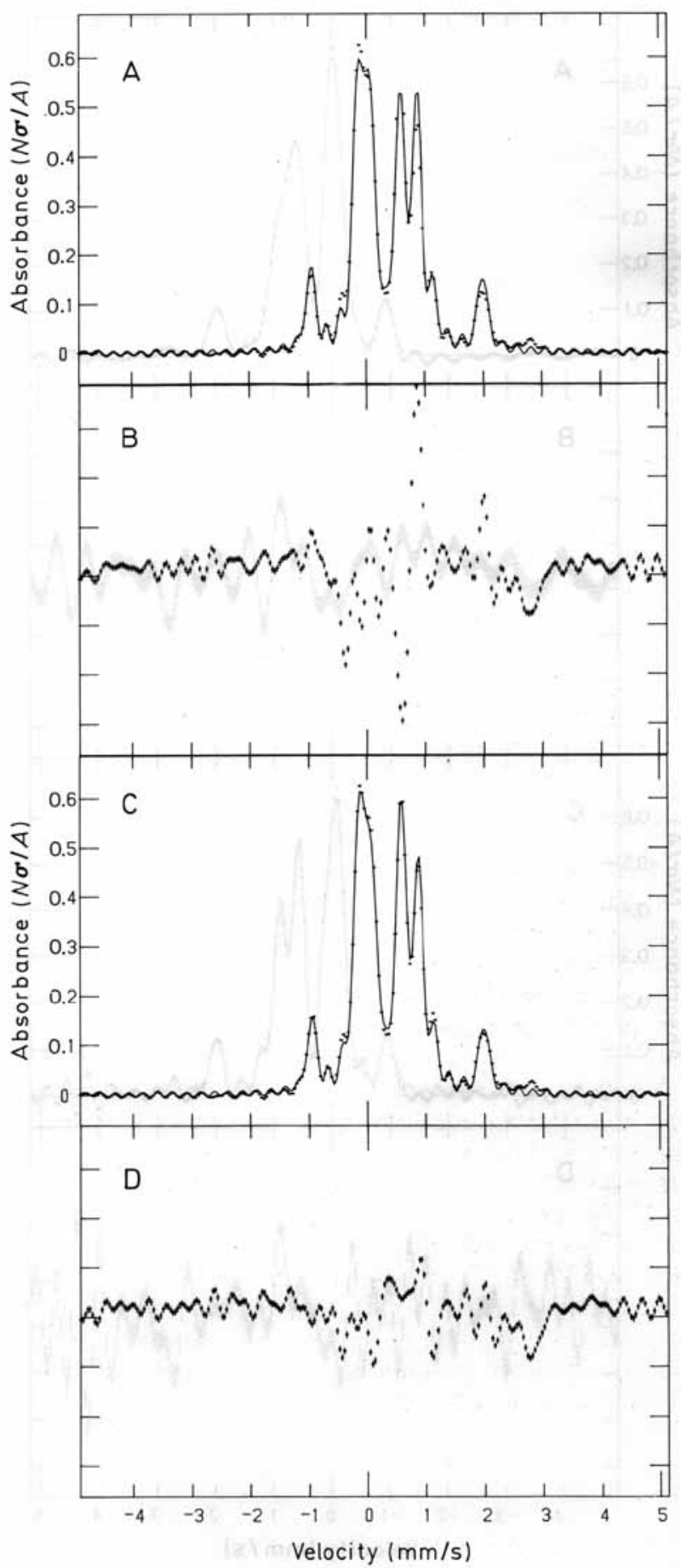

Fig. 2. Mössbauer data and fits for $A v_{1}$, as isolated at $\mathrm{H}=0$ and $125 \mathrm{~K} . \mathrm{Av}_{1}(21.9 \mathrm{mg} / \mathrm{ml})$ was isolated as described in Materials and Methods. (A) Data $(+)$ and fit $(-)$ where iron ratios $\mathrm{Fe}^{2+}: \mathrm{S}: \mathrm{M}: \mathrm{D}$ are $4: 2: 12: 12$. (B) Fit minus data for $\mathrm{A}$, magnified $5 \times$ vertically. (C) Data (+) and fit (-) where ratios are 4:2:16:12. (D) Fit minus data for $\mathrm{C}$, magnified $5 \times$ vertically

integrated total intensity to an error of less than $1 \%$. We expect these numbers to be more accurate than any intensity numbers that we can derive directly from the data because they are less sensitive to baseline error. Both the absorber Debye-Waller factor and the second-order relativistic Doppler shift corrections have been included for the absorber under the Debye model with a Debye temperature of $180 \mathrm{~K}$. 


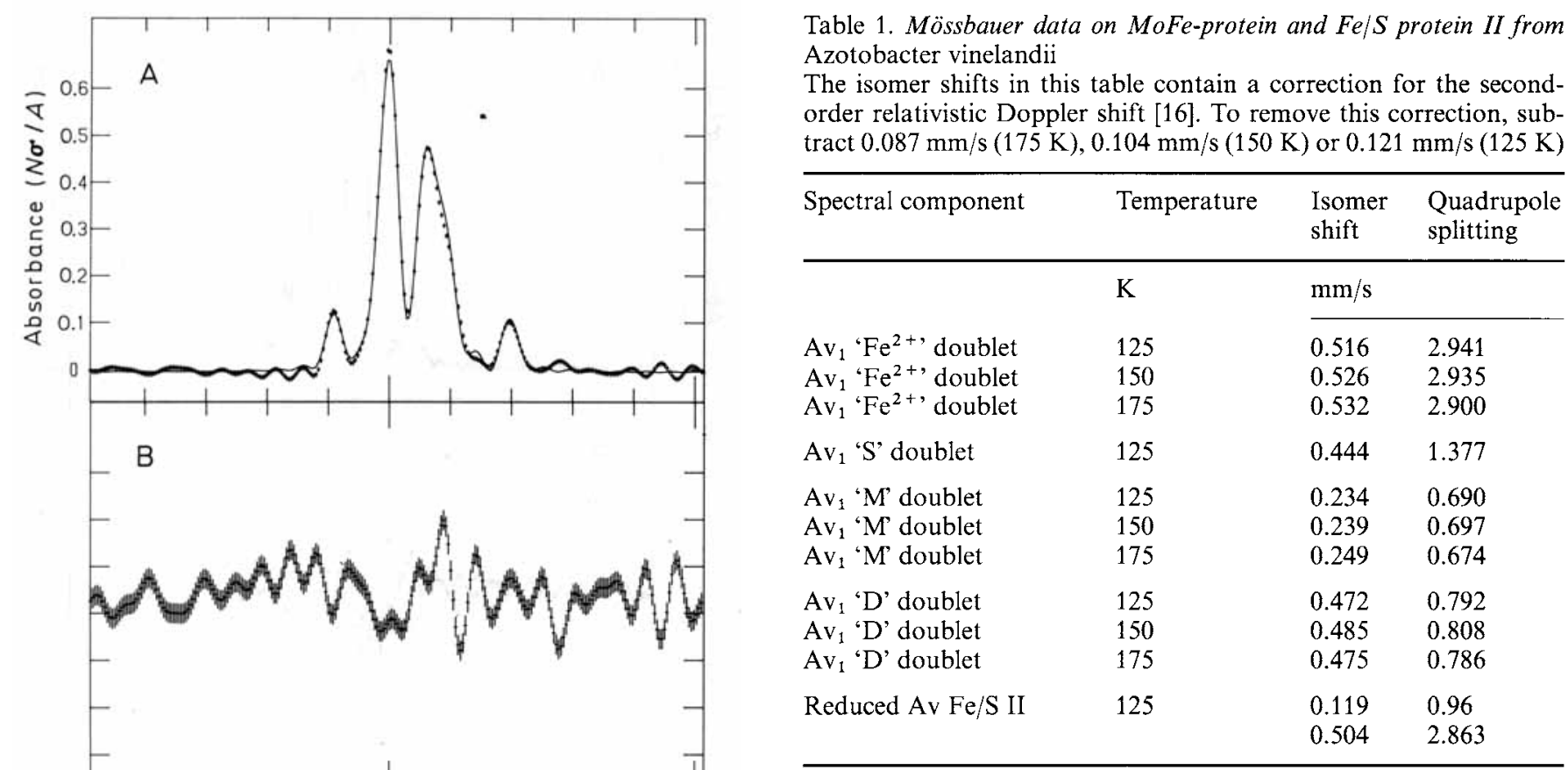

from Debye-Waller factor anomalies and magnetic splittings. In all three fits with this model (Fig. 2C, 3A, 3C) the difference spectra resemble pure noise with the exception of the position of component X, most easily seen in Fig. 3A. We feel that this line is the high-velocity line of a quadrupole doublet $\left(\delta=1.4 \mathrm{~mm} / \mathrm{s}, \Delta E_{\mathrm{q}}=2.8 \mathrm{~mm} / \mathrm{s}\right)$ seen before in the Mössbauer spectra of spinach and Chromatium ferredoxin [25] and some preparations of $\mathrm{Kp}_{2}[6]$. This spectrum is similar to the one from $\mathrm{FeSO}_{4} \cdot 7 \mathrm{H}_{2} \mathrm{O}$ [26] and therefore, probably represents a degradation product between the $\mathrm{S}_{2} \mathrm{O}_{4}^{2-}$ reaction products and a low-pH form of the protein $\mathrm{Fe} / \mathrm{S}$ centers. The maximum height represented by component $\mathrm{X}$ is one-half of an iron atom. Therefore, we do not feel that the presence of $\mathrm{X}$ in these spectra significantly affects the difference spectra.

\section{DISCUSSION}

We have shown in this paper that the $\mathrm{Fe}$ distribution in MoFe-protein can be better explained by a $4: 2: 16: 12$ stoichiometry than by a ratio of $4: 2: 12: 12$ as suggested in the literature. The different stoichiometry is mainly a consequence of our advanced data reduction method since the raw Mössbauer data are not significantly different from those published by others $[1,2,6-9,28]$. We emphasize that our data reduction procedures are most critical when a Mössbauer spectrum has widely varying (weak and strong) line intensities as in the case of reduced MoFe-protein at high temperature. There are several reasons why we think that the problem is as yet unsolved even though the quality of our fits is extremely high. The specific activity of our protein sample was 2250 nmol $\mathrm{C}_{2} \mathrm{H}_{4}$ formed $\cdot \mathrm{min}^{-1} \cdot(\mathrm{mg} \mathrm{Av})^{-1}$ as compared to $1200-1650 \mathrm{nmol} \mathrm{C}_{2} \mathrm{H}_{4}$ formed $\cdot \mathrm{min}^{-1} \cdot \mathrm{mg}^{-1}$ for samples of $\mathrm{Cp}_{1}$ and $\mathrm{Av}_{1}[2,9]$ and $1000-1400 \mathrm{nmol} \mathrm{C}_{2} \mathrm{H}_{4}$ formed $\cdot \min ^{-1} \cdot\left(\mathrm{mg} \mathrm{Kp}_{1}\right)^{-1}[1,6]$. The specific activity of the sample studied here is good; however, samples with a specific activities up to $3000 \mathrm{nmol} \cdot \mathrm{min}^{-1} \cdot \mathrm{mg}^{-1}$ have been obtained [27]. All Mössbauer data of MoFe-proteins are extrapolated to fully active protein with a Mo content of $2 \mathrm{Mo}$ atoms and
Therefore, the agreement in total intensity is simultaneously a demonstration of the precision of the data reduction procedure and a validation of the Debye model over this temperature range. We chose this particular temperature range because our experience has been that in this range proteins follow a Debye model well, that they are too cold to demonstrate the onset of the 'liquid' state $(>200 \mathrm{~K})$, and that they are too warm to show low-temperature complications
Table 1. Mössbauer data on MoFe-protein and Fe/S protein II from

\author{
.
}


$30-36 \mathrm{Fe}$ atoms in a molecule of $220 \mathrm{kDa}$. These extrapolations assume an 'all-or-non' mechanism for metalloss from the MoFe-protein upon inactivation. If MoFe-protein denatures in an 'all-or-none' fashion, then the data in this article represent the fully active in vivo protein; in that case, there are eight iron atoms in the FeMo-cofactor of nitrogenase, not six.

Our interpretation of these-data raises the question: can there by 7 or 9 irons per molybdenum in the cofactor? The answer is yes. The uncertainty in the total iron content of current $\mathrm{Av}_{1}$ preparations $(30-36)$ means that all stoichiometries for the iron clusters are uncertain to one iron atom. However, one of the major points of this paper is to emphasize that some Mössbauer research on nitrogenase is favouring eight irons per FeMo-cofactor rather than the currently accepted number, six. The chemical analysis for the isolated FeMo-cofactor usually gives an iron to molybdenum ratio around $8[10,12]$, except when the isolation procedure includes an iron chelation step [29]. Our tentative conclusion that the FeMo-cofactor in 'the' MoFe-protein always contains $8 \mathrm{Fe}$ per Mo implies that the recent analysis of ' $F$ ' ENDOR from $A v_{1}$ is incomplete [14].

We gratefully acknowledge the contribution of William F. Filter in designing and constructing the Mössbauer spectrometer. One of us (W. R. H.) was supported in part by a fellowship from the European Molecular Biology Organization (ALTF-146-82). We are indebted to R. H. Sands and C. Veeger for their helpful discussions and Miss L. Verstege for typing the manuscript. The present investigation was supported in part by the Netherlands Foundation for Chemical Research (SON) with financial aid from the Netherlands Organization for the Advancement of Pure Research (ZWO) and by NATO grant 1854.

\section{REFERENCES}

1. Smith, B. E. \& Lang, G. (1974) Biochem. J. 137, 169-180.

2. Münck, E., Rhodes, H., Orme-Johnson, W. H., Davis, L. C., Brill, W. J. \& Shah, V. K. (1975) Biochim. Biophys. Acta 400, $32-53$.

3. Mortenson, L. E. \& Thorneley, R. N. F. (1979) Annu. Rev. Biochem. 48, 387-418.

4. Burgess, B. K. (1984) in Advances in nitrogen fixation research (Veeger, C. \& Newton, W. E., eds) pp. 103-114, Nijhoff/Junk/ Pudoc, The Hague.

5. Braaksma, A., Haaker, H. \& Veeger, C. (1983) Eur. J. Biochem. $133,71-76$.

6. Smith, B. E., O’Donnell, M. J., Lang, G. \& Spartalian, K. (1980) Biochem. J. 191, 449-455.
7. Zimmerman, R., Münck, E., Brill, W. J., Shah, V. K., Henzl, M. T., Rawlings, J. \& Orme-Johnson, W. H. (1978) Biochim. Biophys. Acta 537, 185-207.

8. Huynh, B. H., Münck, E. \& Orme-Johnson, W. H. (1979) Biochim. Biophys. Acta 576, 192-203.

9. Huynh, B. H., Henzl, M. T., Christner, J. A., Zimmerman, R., Orme-Johnson, W. H. \& Münck, E. (1980) Biochim. Biophys. Acta $623,124-138$

10. Shah, V. K. \& Brill, W. J. (1977) Proc. Natl Acad. Sci. USA 74, $3249-3253$.

11. Rawlings, J., Shah, V. K., Chrinell, J. R., Brill, W. J., Zimmerman, R., Münck, E. \& Orme-Johnson, W. H. (1978) $J$. Biol. Chem. 253, $1001-1004$.

12. Nelson, M. J., Levy, M. A. \& Orme-Johnson, W. H. (1983) Proc. Natl Acad. Sci. USA 80,147-150.

13. Hoffman, B. M., Roberts, J. E. \& Orme-Johnson, W. H. (1982) J. Am. Chem. Soc. 104, 860-862.

14. Hoffman, B. M., Venters, R. A., Roberts, J. E., Nelson, M. \& Orme-Johnson, W. H. (1982) J. Am. Chem. Soc. 104, 47114712.

15. Orme-Johnson, W. H., Davis, L. C., Henzl, M. T., Averill, B. A., Orme-Johnson, N. R., Münck, E. \& Zimmerman, R. (1977) in Recent developments in nitrogen fixation (Newton, W. E., Postgate, J. R. \& Rodrigues-Barrueco, C., eds) pp. $131-178$, Academic Press, New York.

16. Dunham, W. R., Wu, C. T., Polichar, R. M., Sands, R. H. \& Harding, L. J. (1977) Nucl. Instrum. Methods 145, 537- 553.

17. Filter, W. F. (1983) Ph. D. Thesis, The University of Michigan

18. Clark, L. J. \& Axley, J. H. (1955) Anal. Chem. 27, 2000-2003.

19. Brumby, P. E., Milier, R. W. \& Massey, V. (1965) J. Biol. Chem. 240, 2222-2228.

20. Van de Bogart, M. \& Beinert, H. (1967) Anal. Chem. 20, $325-$ 334.

21. Goa, J. (1953) Scand. J. Clin. Lab. Invest. 5, 218-222.

22. Ure, C. D. M. \& Flinn, P. A. (1971) in Mössbauer effect methodology (Gruverman, I. J., ed.) vol. 7, pp. 245-262, Plenum Press, New York.

23. Oberhettinger, F. (1973) Fourier transforms of distributions and their derivatives, Academic Press, New York.

24. Filter, W. F., Dunham, W. R., Polichar, R. M., Sands, R. H. \& Harding, L. W. (1978) in Frontiers of biological energetics (Dutton, P. L., Leigh, J. S. \& Scarpa, A., eds) vol. 1, pp. $603-$ 616, Academic Press, New York

25. Moss, T. H., Bearden, A. J., Bartsch, R. G., Cusanovich, M. A. \& San Pietro, A. (1968) Biochemistry 7, 1591-1596.

26. Flick, E., Kerler, W. \& Neuwirth, W. (1963) Angew. Chem. 75, $461-488$.

27. Burgess, B. K., Jacobs, D. B. \& Stiefel, E. I. (1980) Biochim. Biophys. Acta 614, 196-209.

28. Kelly, M. \& Lang, G. (1970) Biochim. Biophys. Acta 223, 86104.

29. Yang, S.-S., Pan, W.-H., Friesen, G. D., Burgess, B. K., Corbin, J. L., Stiefel, E. I. \& Newton, W. E. (1982) J. Biol. Chem. 257, $8042-8048$

W. R. Dunham and W. R. Hagen, Biophysics Research Division, Institute of Science and Technology, University of Michigan, Ann Arbor, Michigan, USA 48109

A. Braaksma and H. Haaker, Laboratorium voor Biochemie der Landbouwhogeschool, De Dreijen 11, NL-6703-BC Wageningen, The Netherlands

H. J. Grande, Instituut voor Physische Chemie, Nederlandse Organisatie voor Toegepast-Natuurwetenschappelijk Onderzoek, Postbus 198, NL-3700-AC Zeist, The Netherlands 